\title{
DIAGNÓSTICOS DE ENFERMAGEM ENTRE USUÁRIOS DE ANTICOAGULANTE ORAL ACOMPANHADOS EM AMBULATÓRIO
}

\author{
NURSING DIAGNOSES AMONG USERS OF ORAL \\ ANTICOAGULANTS IN OUTPATIENT FOLLOW-UP
}

\section{DIAGNÓSTICOS DE ENFERMERÍA ENTRE USUARIOS DE ANTICOAGULANTE ORAL ACOMPAÑADOS EN AMBULATORIO}

\author{
Daiana de Freitas ${ }^{1}$ \\ Kelly Ribeiro \\ João Lucas Campos de Oliveira ${ }^{2}$ \\ Fabiana Gonçalves de Oliveira Azevedo Matos ${ }^{3}$ \\ Ariana Rodrigues da Silva Carvalho ${ }^{4}$ \\ Cláudia Ross ${ }^{5}$
}

\begin{abstract}
Como citar este artigo: Freitas D, Ribeiro K, Oliveira JLC, Matos FGOA, Carvalho ARS, Ross C. Diagnósticos de enfermagem entre usuários de anticoagulante oral acompanhados em ambulatório. Rev baiana enferm 2017;31(3):e20356.

Objetivo: identificar o perfil sociodemográfico e clínico, bem como principais diagnósticos de enfermagem entre usuários de anticoagulantes de uso oral acompanhados em ambulatório. Método: pesquisa descritiva, transversal de abordagem quantitativa. Realizada com 128 usuários elegíveis acompanhados em ambulatório de hospital universitário público do Paraná, Brasil. Foram coletadas, em consulta de enfermagem e fonte documental, variáveis sociodemográficas e clínicas, bem como identificados diagnósticos de enfermagem segundo taxonomia da NANDAInternational. Resultados: a amostra foi predominantemente idosa; casada; de baixa escolaridade; com indicação de anticoagulação oral por fibrilação atrial e trombose venosa profunda; com adequado tempo médio de coagulação, expresso pela Razão Normalizada Internacional. Os cinco diagnósticos de enfermagem elencados pertenciam às classes de respostas cardiovasculares/pulmonares; infecção; ingestão; e controle da saúde. Conclusão: os diagnósticos de enfermagem identificados coadunam-se ao perfil clínico dos usuários de anticoagulantes de uso oral acompanhados em ambulatório, o que reflete um cenário mais focado nas questões orgânico-funcionais, ainda que a necessidade de melhor controle da própria saúde e de domínio da promoção de saúde tenham emergido em menor proporção.
\end{abstract}

Descritores: Diagnóstico de enfermagem. Anticoagulantes. Ambulatório hospitalar. Processos de enfermagem.

\footnotetext{
Enfermeiras. Especialistas, na modalidade de Residência, em Gerenciamento de Enfermagem em Clínica Médica e Cirúrgica pela Universidade Estadual do Oeste do Paraná. Cascavel, Paraná, Brasil.

Enfermeiro. Mestre. Doutorando no Programa de Pós-graduação em Enfermagem da Universidade Estadual de Maringá. Docente colaborador dos cursos de Graduação em Enfermagem e Residência em Gerenciamento de Enfermagem em Clínica Médica e Cirúrgica da Universidade Estadual do Oeste do Paraná. Cascavel, Paraná, Brasil.enfjoaolcampos@yahoo.com.br

3 Enfermeira. Doutora em Ciências. Professora adjunta ao Colegiado de Enfermagem da Universidade Estadual do Oeste do Paraná. Cascavel, Paraná, Brasil.

Enfermeira. Doutora em Enfermagem Fundamental. Professora adjunta do Colegiado de Enfermagem da Universidade Estadual do Oeste do Paraná. Cascavel, Paraná, Brasil.

5 Enfermeira. Doutora em Microbiologia. Professora associada ao Colegiado de Enfermagem da Universidade Estadual do Oeste do Paraná. Cascavel, Paraná, Brasil.
} 
Objective: to identify the sociodemographic and clinical profile, as well as the main nursing diagnoses among users of oral anticoagulants in outpatient follow-up. Method: it was a descriptive cross-sectional study with a quantitative approach, conducted with 128 eligible users in outpatient follow-up at a public university hospital in Paraná, Brazil. Sociodemographic and clinical variables were collected in nursing consultations and documentary sources, and the nursing diagnoses were identified according to NANDA International taxonomy. Results: the sample was composed predominantly of elderly people; married; with low schooling; with indication of oral anticoagulation for atrial fibrillation and deep venous thrombosis; and adequate average clotting time expressed by the International Normalized Ratio. The five nursing diagnoses listed belonged to the classes of Cardiovascular/Pulmonary responses; Infection; Ingestion; and Health management. Conclusion: the nursing diagnoses identified combine with the clinical profile of the users of oral anticoagulants in outpatient follow-up, thus reflecting a scenario more focused on organic-functional issues, although the need for better control of one's own bealth and domain of health promotion have emerged in a lesser extent.

Descriptors: Nursing Diagnosis. Anticoagulants. Outpatient Clinics, Hospital. Nursing Process.

Objetivo: identificar el perfil sociodemográfico y clínico, así como los principales diagnósticos de enfermería entre usuarios de anticoagulantes de uso oral acompañados en ambulatorio. Método: investigación descriptiva, transversal con enfoque cuantitativo. Realizada con 128 usuarios elegibles acompañados en ambulatorio del hospital universitario público de Paraná, Brasil. Fueron recolectadas, en consulta de enfermería y base documental, variables sociodemográficas y clínicas, además de la identificación de diagnósticos de enfermería, de acuerdo con la taxonomía de la NANDA-International. Resultados: la muestra se compuso predominantemente de personas mayores; casadas; de baja escolaridad; con indicación de anticoagulación oral por fibrilación atrial y trombosis venosa profunda; con una media de tempo adecuado de coagulación, expresado a través de la Razón Normalizada Internacional. Los cinco diagnósticos de enfermería enumerados pertenecian a las clases de respuestas cardiovasculares/pulmonares; infección; ingestión y; control de la salud. Conclusión: los diagnósticos de enfermería identificados se coadunan al perfil clinico de los usuarios de anticoagulantes de uso oral acompañados en ambulatorio, lo que refleja un escenario más orientado para las cuestiones orgánico-funcionales, a pesar de que a necesidad de un mejor control de la propia salud y del dominio de la promoción de la salud hayan emergido en menor proporción.

Descriptores: Diagnóstico de enfermería. Anticoagulantes. Ambulatorio hospitalario. Procesos de enfermería.

\section{Introdução}

Os anticoagulantes orais (ACO), denominados de anti-vitamina $\mathrm{K}$, também conhecidos como agentes cumarínicos, possuem a capacidade de reduzir a coagulação sanguínea, interferindo na hemostasia. Por isso, são capazes de atuar quando há lesão do endotélio, contendo o sangramento ${ }^{(1)}$.

Os principais antagonistas da vitamina $\mathrm{K}$ aprovados para o uso clínico no Brasil incluem a varfarina sódica e a femprocumona, que estão indicados nas doenças/situações de importância clínica e epidemiológica em condições como fibrilação atrial (FA), trombose venosa profunda (TVP), embolia pulmonar, trombofilias, prevenção de embolia arterial sistêmica em usuários de válvulas cardíacas, acidente vascular cerebral e infarto recorrente ${ }^{(2)}$.

A utilização de ACO deve ser extremamente criteriosa, pois essa decisão precisa considerar os benefícios para a prevenção de embolismo e os riscos potenciais de indução hemorrágica ${ }^{(1-2)}$. Diante desses riscos, é necessário o monitoramento laboratorial rigoroso, com correção da dose do anticoagulante, utilizando-se de testes laboratoriais como o Tempo de Protrombina (TP), expresso pela Razão Normalizada Internacional $(\mathrm{RNI})^{(2)}$.

Os anticoagulantes antivitamina $\mathrm{K}$ possuem algumas limitações para sua utilização, como a janela terapêutica estreita, imprevisibilidade de sua farmacodinâmica e farmacocinética, fatores individuais, genéticos e ambientais ${ }^{(2-3)}$. Neste sentido, é importante a presença de uma equipe interdisciplinar capacitada para a promoção e manutenção da saúde de indivíduos em uso de anticoagulantes orais, incluindo aqueles em acompanhamento ambulatorial, tendo em vista a necessidade de controle de agravos, prevenção de danos e promoção de qualidade de vida ${ }^{(1)}$. 
Dentre os membros da equipe de saúde, há de se destacar o papel do enfermeiro, visto que o profissional, historicamente, posiciona a gerência com foco na viabilização de estratégias racionais de assistência ${ }^{(4)}$. Na gerência do cuidado, emerge a importância da Sistematização da Assistência de Enfermagem (SAE), que pode ser compreendida como uma ferramenta de trabalho que proporciona melhoria da qualidade da assistência prestada ao indivíduo por meio do planejamento, execução e avaliação das ações de interesse da equipe de enfermagem ${ }^{(5-6)}$. Além do cuidado direto, a SAE é importante para direcionar ações de gestão de pessoas, fator inegável à dimensão gerencial do trabalho do enfermeiro $^{(7)}$.

O Processo de Enfermagem (PE), dentre os elementos da SAE, possibilita o desenvolvimento de um método científico no trabalho para a gestão do cuidado pelo enfermeiro ${ }^{(5-7)}$. Por isso, o PE constituído por cinco etapas - coleta de dados/histórico de enfermagem, diagnóstico de enfermagem, planejamento, implementação e avaliação de intervenções de enfermagem - é um método de trabalho sistemático que norteia o cuidado profissional de enfermagem ${ }^{(7-8)}$.

A identificação dos diagnósticos de enfermagem (DE) consiste num processo de julgamento clínico ${ }^{(9-10)}$. Destarte, os DE estruturam o conhecimento e buscam a definição do papel e do domínio da enfermagem, ao mesmo tempo em que auxiliam na avaliação da assistência prestada, direcionam o cuidado, promovem o ensino, definem as funções da equipe de enfermagem, além de estimularem a participação do usuário no seu tratamento ${ }^{(10)}$.

Dentre as maneiras de formular e nominar os diagnósticos de enfermagem, um sistema muito utilizado na prática clínica em todo o mundo foi desenvolvido pela Associação Norte-Americana de Diagnósticos de Enfermagem (NANDA-International), sendo adotado pela associação das enfermeiras norte-americanas como a metodologia oficial de taxonomia de diagnósticos para os Estados Unidos da América, desde a década de $1980^{(11)}$.
Conhecer o perfil de saúde e os diagnósticos de enfermagem de uma clientela específica favorece a construção de uma prática voltada para suas reais necessidades ${ }^{(9-10)}$. Em outras palavras, somar o conhecimento das características de uma dada população, atrelando a identificação diagnóstica, tende a ser um meio de o enfermeiro racionalizar o processo de cuidado de forma respaldada. Apesar disso, o conhecimento que abrange a sistematização da assistência nesse tipo peculiar de clientela ainda é incipiente, com muitas lacunas a serem preenchidas. Com base nas considerações expostas, questionou-se: Quais são os principais diagnósticos de enfermagem identificados junto aos usuários em tratamento de anticoagulação oral acompanhados em ambulatório?

A fim de sanar a pergunta enunciada, este estudo objetiva identificar o perfil sociodemográfico e clínico, bem como os principais diagnósticos de enfermagem entre os usuários de anticoagulantes de uso oral acompanhados em ambulatório. Para tanto, espera-se fornecer subsídios para a qualificação da assistência prestada aos indivíduos nessa modalidade terapêutica, contribuindo para a sua qualidade de vida e adesão ao longo do tratamento.

\section{Método}

Trata-se de um estudo descritivo, de delineamento transversal e abordagem quantitativa. Foi desenvolvido no Ambulatório de Anticoagulação Oral de um hospital universitário público do interior do estado do Paraná, Brasil, que possui capacidade operacional de 210 leitos vinculados exclusivamente ao Sistema Único de Saúde (SUS). Por sua vez, o ambulatório campo de estudo é referência para acompanhamento de pacientes que estiveram ou não internados no hospital, sendo utilizado para tratamento de usuários das mais diversas especialidades médicas, clínicas e cirúrgicas, bem como inclui atendimento do enfermeiro em todas as áreas.

Participaram deste estudo indivíduos que atenderam aos seguintes critérios de elegibilidade: usuários com indicação para o uso de 
ACO por qualquer etiologia, maiores de 18 anos, de ambos os sexos, e assíduos nas consultas de enfermagem durante o recorte temporal de pesquisa, que compreendeu o período de fevereiro a junho de 2013. Pacientes que porventura não tivessem condições clínicas de resposta verbal ou motora para seu consentimento e estivessem na ausência de um familiar responsável durante as consultas de enfermagem seriam excluídos. Entretanto, não houve nenhuma exclusão por esse critério, porque a participação no estudo também estava condicionada à anuência de cada participante ou acompanhante, mediante leitura e assinatura do Termo de Consentimento Livre e Esclarecido (TLCE).

Para a coleta de dados, utilizou-se um instrumento de caracterização sociodemográfica e clínica dos usuários adaptado de outra versão utilizada em estudos que também envolveram pacientes em tratamento de anticoagulação oral $^{(12-14)}$. Tal instrumento foi construído e validado (validade de face, de conteúdo e validade semântica) em estudos anteriores ${ }^{(12-14)}$. O instrumento usado contemplava a extração, por entrevista com o paciente em consulta de enfermagem e fonte documental, das seguintes variáveis: sexo, idade, escolaridade, estado civil, ocupação, droga de uso na ACO, comorbidades, complicações durante o tratamento de ACO e necessidade de internação hospitalar pela complicação.

Ademais, alguns dados clínicos (indicação clínica de uso de ACO e exames laboratoriais de tempo de coagulação sanguínea) foram obtidos em fonte documental - e registrados também no instrumento de coleta de dados - que se valeu dos prontuários impressos e eletrônicos, com base em software terceirizado pelo hospital. Na tentativa de evitar classificações equivocadas dos sujeitos que apresentassem (ou não) alterações da coagulação sanguínea evidenciadas apenas no valor da última coleta da RNI, calculou-se a média desses valores, com base nos dados do último ano de tratamento, independentemente do número de amostras que tivessem sido realizadas e do tempo que o indivíduo estivesse em seguimento ambulatorial.
Para a classificação dos DE utilizou-se o sistema desenvolvido pela NANDA-International, com o estabelecimento da taxonomia diagnóstica, bem como os respectivos domínios e classes de cada DE levantado ${ }^{(11)}$. A identificação dos DE deu-se no momento da consulta individual com os participantes, realizada concomitantemente por duas enfermeiras residentes em Gerenciamento de Enfermagem em Clínica Médica e Cirúrgica, no desenvolvimento de seu treinamento em serviço no ambulatório. As pesquisadoras foram previamente treinadas para tal fim por duas docentes do programa de Residência, enfermeiras, doutoras, atuantes nas áreas de Enfermagem em Cardiologia e Sistematização da Assistência de Enfermagem, respectivamente.

Para processamento e análise dos dados foi utilizado o programa Statistical Package for the Social Sciencies (SPSS), versão 21.0. Os dados tabulados possibilitaram a análise descritiva em medidas de proporção em percentual para as variáveis categóricas, e de tendência central (mediana e média) e variabilidade (desvio-padrão) para as variáveis contínuas.

Foram respeitados todos os preceitos éticos exigidos pela Resolução do Conselho Nacional de Saúde n. 466/2012. Dessa forma, o projeto de pesquisa foi submetido e aprovado pelo Comitê de Ética em Pesquisa com Seres Humanos da Universidade Estadual do Oeste do Paraná (UNIOESTE), sob Parecer n. 201/2010.

\section{Resultados}

No total, 236 pessoas estavam cadastradas no Ambulatório de Anticoagulação oral. Entretanto, 108 não participaram do estudo, por não atenderem aos critérios de inclusão, a saber: menores de 18 anos de idade $(n=3)$; abandono do tratamento $(n=4)$; recusa de participação na pesquisa $(n=7)$; óbito $(n=9)$; consulta agendada posteriormente ao recorte temporal de pesquisa $(n=10)$; mudança de acompanhamento para outro serviço ( $n=14)$; alta médica $(n=23)$; e não localizados $(n=38)$.

Ante o exposto, o estudo contou com uma amostra analisada de 128 participantes. 
A Tabela 1 ilustra os achados de caracterização da amostra, segundo variáveis sociodemográficas de sexo, idade, estado civil, escolaridade e ocupação.

Tabela 1 - Características sociodemográficas dos usuários de anticoagulação oral acompanhados em ambulatório. Cascavel, Paraná, Brasil - 2013. (n=128)

\begin{tabular}{|c|c|c|c|c|c|}
\hline Variáveis & $\mathbf{n}$ & $\%$ & Mediana & Amplitude & $\begin{array}{c}\text { Média } \\
\text { (Desvio Padrão) }\end{array}$ \\
\hline \multicolumn{6}{|l|}{ Sexo } \\
\hline Feminino & 66 & 51,6 & & & \\
\hline Masculino & 62 & 48,4 & & & \\
\hline Idade (anos) & & & 65,5 & 21 a 89 & $62,2(14,1)$ \\
\hline Até 40 anos & 11 & 8,6 & & & \\
\hline 41 a 59 anos & 34 & 26,6 & & & \\
\hline Acima de 60 anos & 83 & 64,8 & & & \\
\hline \multicolumn{6}{|l|}{ Estado civil } \\
\hline Casado/União consensual & 83 & 64,8 & & & \\
\hline Viúvo & 21 & 16,4 & & & \\
\hline Solteiro & 16 & 12,5 & & & \\
\hline Separado & 5 & 3,9 & & & \\
\hline Outros & 3 & 2,3 & & & \\
\hline Escolaridade* & & & 4,0 & 0 a 17 & $4,7(3,9)$ \\
\hline Analfabeto & 20 & 15,6 & & & \\
\hline 1 a 4 & 58 & 45,3 & & & \\
\hline 5 a 8 & 32 & 25,0 & & & \\
\hline 9 a 11 & 10 & 7,8 & & & \\
\hline Mais de 11 & 8 & 6,3 & & & \\
\hline \multicolumn{6}{|l|}{ Ocupação } \\
\hline Aposentado/Pensionista & 55 & 43,0 & & & \\
\hline Com atividades remuneradas & 45 & 35,1 & & & \\
\hline Sem atividades remuneradas & 28 & 21,9 & & & \\
\hline
\end{tabular}

Fonte: Elaboração própria.

* Em anos de estudo formal.

Quanto aos dados clínicos, a fibrilação atrial $(34,4 \%)$ foi a principal indicação ao uso de ACO, seguida da trombose venosa profunda (19,5\%) e da prótese valvar cardíaca mecânica $(17,2 \%)$. A varfarina sódica $(89,1 \%)$ foi o ACO mais usado entre os participantes do estudo, seguida da femprocumona $(10,9 \%)$. Outras morbidades foram identificadas entre os participantes do estudo, além daquela que indicou o uso do ACO, obtendo-se média de 2,9 morbidades, variando de zero a oito. Dentre as comorbidades mais observadas estava a hipertensão arterial sistêmica (62,5\%), seguida da insuficiência cardíaca $(28,9 \%)$.

No que se refere ao valor do tempo da coagulação sanguínea expresso pelo valor de RNI, os participantes do estudo apresentaram uma média de 2,2 ( $\mathrm{DP}=0,4)$, variando de 1,03 a 4,18, permanecendo a média de $40 \%$ do tempo dentro do limite terapêutico esperado para o tratamento Time-in-Therapeutic Range (TTR) ${ }^{(1-2)}$.

Dentre os usuários de ACO, 37 (28,9\%) apresentaram complicações no decorrer do tratamento. Dentre estes 27 (73\%) manifestaram evento hemorrágico e 10 (27\%) evento trombótico. Destes, 23 (18\%) necessitaram de internação hospitalar.

Entre os usuários que interromperam a terapia $(n=35)$ com ACO $(27,3 \%)$, os principais motivos foram: a necessidade de submeter-se a procedimentos invasivos (17; 48,6\%); presença de algum tipo de complicação (7; 20\%); interrupção por conta própria $(7 ; 20 \%)$ e RNI aumentado, apesar de não ter evidenciado complicação 
(4; 11,4\%). Desta totalidade, $25(71,4 \%)$ dos sujeitos interromperam a terapia do ACO mediante orientação médica.

Indo ao encontro do objetivo proposto, os dados obtidos neste estudo permitiram identificar cinco diagnósticos de enfermagem, distribuídos em quatro domínios e quatro classes da taxonomia II da NANDA-I ${ }^{(11)}$. Neste aspecto, os achados relativos aos diagnósticos de enfermagem e seus respectivos domínios e classes estão apresentados em ordem decrescente de frequência na Tabela 2.

Tabela 2 - Diagnósticos de Enfermagem, domínios e classes diagnósticas segundo a Taxonomia II da NANDA-International entre usuários de anticoagulação oral acompanhados em ambulatório. Cascavel, Paraná, Brasil - 2013. (n=128)

\begin{tabular}{|c|c|c|c|c|}
\hline Diagnóstico & Domínio & Classe & $\mathbf{n}$ & $\%$ \\
\hline Risco de sangramento & $\begin{array}{l}\text { Domínio } 4 \text { Atividade/ } \\
\text { Repouso }\end{array}$ & $\begin{array}{l}\text { Respostas cardiovasculares/ } \\
\text { pulmonares }\end{array}$ & 128 & 100 \\
\hline Risco de infecção & $\begin{array}{l}\text { Domínio } 11 \text { Segurança/ } \\
\text { Proteção }\end{array}$ & Infecção & 128 & 100 \\
\hline $\begin{array}{l}\text { Nutrição desequilibrada: } \\
\text { mais do que as necessidades } \\
\text { corporais }\end{array}$ & $\begin{array}{l}\text { Domínio } 2 \\
\text { Nutrição }\end{array}$ & Ingestão & 90 & 70,3 \\
\hline $\begin{array}{l}\text { Perfusão tissular periférica } \\
\text { ineficaz }\end{array}$ & $\begin{array}{l}\text { Domínio } 4 \text { Atividade/ } \\
\text { Repouso }\end{array}$ & $\begin{array}{l}\text { Respostas cardiovasculares/ } \\
\text { pulmonares }\end{array}$ & 43 & 33,6 \\
\hline $\begin{array}{l}\text { Autocontrole ineficaz da } \\
\text { saúde }\end{array}$ & $\begin{array}{l}\text { Domínio } 1 \\
\text { Promoção da Saúde }\end{array}$ & Controle da Saúde & 7 & 20 \\
\hline
\end{tabular}

Fonte: Elaboração própria.

\section{Discussão}

Com relação às características sociodemográficas dos sujeitos da pesquisa, pode-se afirmar que houve concordância com os resultados de outros estudos envolvendo o mesmo tipo de população ${ }^{(14-16)}$, com discreta predominância do sexo feminino (51,6\%); maior número de pacientes na faixa etária acima de 60 anos de idade; casados ou vivendo em união consensual (64,8\%); predominância de aposentados ou pensionistas (43\%); e baixa escolaridade (45,3\%).

O nível de escolaridade é um fator que pode prejudicar o entendimento dos indivíduos quanto à terapia medicamentosa estabelecida, a exemplo do uso dos $\mathrm{ACO}^{(15)}$. O estudo apontou que a maioria dos sujeitos pesquisados tinha baixa escolaridade, o que é comum nas instituições de saúde que atendem majoritariamente pelo SUS ${ }^{(12)}$, reforçando a ideia de que, não somente nesses serviços, o enfermeiro deve posicionar-se como gerente do cuidado por meio, inclusive, do empoderamento dos sujeitos acerca de sua situação de saúde e intervenções propostas.

As indicações mais frequentes para o uso de ACO foram a FA $(34,4 \%)$, dado que também foi observado em outros estudos com pacientes submetidos à mesma modalidade terapêutica, assim como a TVP $(19,5 \%)^{(15-16)}$. A varfarina sódica foi o ACO mais prescrito para essa população (89,1\%), o que também foi verificado em outros estudos $^{(14-16)}$. Isso possivelmente significa que a delimitação epidemiológica e terapêutica bem definida entre usuários de ACO é um fator disseminado em diferentes contextos, o que tende a facilitar prática sistematizada do enfermeiro em prol da qualidade da assistência no contexto das orientações quanto às possíveis reações medicamentosas e boas práticas para correta adesão.

Foram identificados cinco diagnósticos de enfermagem para os usuários de ACO que frequentam o ambulatório em estudo. Destaca-se que o DE Risco de sangramento, definido pela NANDA-International como "risco de redução no volume de sangue capaz de comprometer a 
saúde"(11) esteve presente nos 128 (100\%) indivíduos que compuseram a amostra, o que já era esperado, porque o uso de medicamentos anticoagulantes é, sabidamente, um fator de risco clínico para sangramento ${ }^{(1-2)}$. Apesar disso, as impressões diagnósticas do enfermeiro merecem destaque, visto que direcionam as práticas de cuidado, indo além da constatação "meramente" clínica, bem como as avaliações do resultado da assistência.

A complicação hemorrágica é a mais temida, quando se trata de anticoagulação oral, haja vista que são substâncias que impedem a formação de trombos, mas a sua dose ideal, para que o indivíduo permaneça dentro do limite terapêutico desejado, é bastante tênue, podendo evoluir para quadros de sangramentos que podem ser letais, do tipo: gengivorragia; epistaxe; hematúria; sangramento excessivo em cortes simples; hematomas e ainda sangramentos em órgãos internos, como hemorragias digestivas ${ }^{(17-18)}$.

Os idosos estão entre os usuários que mais se beneficiam da terapêutica com ACO, no entanto também são aqueles com maior risco de complicações hemorrágicas $^{(1)}$. Desse modo, nota-se, nos achados deste estudo, que os indivíduos que apresentaram complicações hemorrágicas (70,4\%) estavam na faixa etária acima de 60 anos, fato que também reforça a necessidade de supervisão rigorosa da equipe de saúde quanto aos cuidados de acompanhamento. No contexto do enfermeiro, vale destacar que, como ator protagonista no direcionamento do cuidado, ele deve orientar o idoso e seus familiares à prevenção de quedas, cortes, lesões, entre outros fatores que podem desencadear uma reação hemorrágica.

Os eventos hemorrágicos podem atingir de 4,2 a $15 \%$ dos indivíduos que fazem uso de ACO e este fato pode estar relacionado ao inadequado emprego dessa medicação ${ }^{(17)}$. Também por esse motivo, faz-se necessária a ação rápida da equipe de enfermagem em verificar sinais de sangramento para agir nos momentos iniciais, bem como repassar informações para os indivíduos e familiares a respeito dos sinais e sintomas de sangramento, a fim de evitar complicações mais graves ${ }^{(14,17)}$.
O valor da RNI é um dos parâmetros usados para avaliar se a terapia anticoagulante oral está sendo efetiva ${ }^{(1-3)}$. Os valores da RNI almejados, de maneira geral, variam de acordo com a indicação para o uso do ACO, isto é, deveriam variar entre 2,0 e 3,0, para aqueles indivíduos com trombose venosa profunda, com fibrilação atrial isolada ou associada à doença valvar reumática, ainda não operados, ou com prótese modelo biológico, independentemente da posição; e valores entre 2,5 e 3,5 para os indivíduos em uso de prótese valvar cardíaca mecânica, independentemente da posição - mitral ou aórtica - e do ritmo cardíaco ${ }^{(3)}$. Considerando esse indicativo para os participantes do presente estudo, o valor da RNI variou de 1,03 a 4,18, com média de 2,2, indo ao encontro das recomendações, apesar da amplitude da variância dessa medida.

Um intervalo terapêutico único para o uso de ACO pode não ser ideal para todas as indicações, entretanto uma anticoagulação de moderada intensidade $(\mathrm{RNI}=2,0-3,0)$ é efetiva para a maioria das indicações clínicas ${ }^{(17)}$, o que corrobora a interpretação de que, em acordo com a avaliação da RNI dos sujeitos estudados, eles podem ser considerados dentro da faixa terapêutica esperada.

Vale ressaltar que, apesar de os sujeitos terem sido avaliados como dentro da faixa terapêutica para anticoagulação oral, houve uma variação dos valores da RNI para cima e para baixo do almejado, podendo causar estado de hipo ou hipercoagulabilidade, confirmando dados de estudo realizado em um hospital terciário do interior de São Paulo ${ }^{(12)}$. Destarte, sabe-se que manter os valores da RNI dentro do limite terapêutico desejado é um desafio para a equipe cuidadora e para o usuário em terapia por ACO, mas, sem dúvida, um fator a ser almejado constantemente, o que inclui o controle laboratorial e a própria coleta de dados com o paciente e/ou familiar/acompanhante ${ }^{(1)}$.

Dentre os participantes do estudo, 73\% manifestaram complicações hemorrágicas, ocorrência corroborada na literatura correlata às pesquisas com usuários de $\mathrm{ACO}^{(12-14)}$. Apesar da incidência das complicações hemorrágicas ou 
tromboembólicas não ser homogênea na literatura, as complicações hemorrágicas são mais comuns e as mais temidas durante a terapêutica com ACO, pois podem ser fatais ${ }^{(17)}$. Isso reforça o achado relativo ao diagnóstico de enfermagem de risco de sangramento presente na totalidade da amostra (Tabela 2), enfatizando a importância de o enfermeiro utilizar o raciocínio clínico e também a taxonomia diagnóstica de sua escolha como fatores promotores de visibilidade no seu trabalho, no contexto do direcionamento do cuidado.

Outro diagnóstico de enfermagem identificado em 100\% dos sujeitos da pesquisa foi o Risco de infecção, definido pela NANDA-International como "risco de ser invadido por organismos patogênicos"(11). Destaca-se que este diagnóstico não foi evidenciado devido ao uso do ACO propriamente dito, mas foi relacionado à necessidade de coletas de sangue venoso frequentes para verificação do valor de RNI, que é uma demanda obrigatória para essa população ${ }^{(1)}$. Por esse motivo, o enfermeiro deve militar pelo cumprimento da assepsia na coleta de material biológico, capacitando a equipe, quando necessário, e utilizando-se racionalmente das tecnologias disponíveis ao cuidado.

A inadequada preparação da pele antes da realização de procedimentos invasivos pode contribuir sobremaneira para o desenvolvimento de infecções ${ }^{(19)}$. Embora a realização da punção venosa periférica seja considerada uma técnica simples e corriqueira na prática assistencial de enfermagem, ela demanda importantes processos decisórios quanto à escolha do melhor local para punção, o uso de materiais apropriados e execução da técnica adequada ${ }^{(19-20)}$. Recomenda-se a utilização de cateteres com menor calibre com a finalidade de evitar flebites mecânicas e, consequentemente, uma infecção de corrente sanguínea ${ }^{(20)}$. Entretanto, a avaliação individualizada de cada paciente precisa ser feita ${ }^{(19-20)}$, o que requer o papel do enfermeiro.

No pensamento de escolha de melhores condições para a assistência ao paciente, cumpre aqui uma reflexão acerca da identificação em 100\% da amostra de dois rótulos diagnósticos voltados ao risco: o risco para sangramento e para infecção. Esta reflexão é necessária, pois, em tese, um diagnóstico de enfermagem serve para individualizar o cuidado e direcionar ações mais condizentes com a realidade singular de cada ser humano ${ }^{(10-11)}$. Logo, ainda que o resultado seja reflexo de uma pesquisa e não somente de práticas clínicas executadas com os pacientes participantes, massificar dois diagnósticos de enfermagem para todos os usuários pode ser preocupante no intuito de fragilizar o potencial individualizante dos diagnósticos de enfermagem.

Ante o exposto, vai-se além na reflexão proposta sobre a ideia de que, para individualizar o cuidado de fato, o enfermeiro, além de utilizar racional e corretamente as taxonomias diagnósticas para o planejamento assistencial, também precisa incorporar criticamente os meios e instrumentos do trabalho na gerência da assistência ${ }^{(5)}$. Por exemplo, em relação ao risco para sangramento identificado entre os diagnósticos de enfermagem, os enfermeiros do ambulatório podem usar os resultados de exames laboratoriais para definir mais clara e direcionadamente quais os pacientes mais expostos a riscos dessa ordem e não se "limitar" à identificação rotular do diagnóstico de enfermagem.

O diagnóstico de enfermagem Nutrição desequilibrada mais que as necessidades corporais, definido pela NANDA-International como "ingestão de nutrientes que excede as necessidades metabólicas"(11), foi identificado em 90 (70,3\%) indivíduos que frequentavam o Ambulatório de Anticoagulação Oral em questão. Destes, 21 $(23,1 \%)$ tinham a RNI fora da faixa terapêutica, isto é, abaixo de 2,0 ou acima de 3,0. O Índice de Massa Corporal (IMC) acima dos limites desejáveis pode comprometer a terapêutica estabelecida, visto que o ganho de peso e até mesmo a obesidade, enquanto comorbidade, é um fator importante para a redução do efeito anticoagulante ${ }^{(17)}$. Este é um fator que tende a salientar o fortalecimento da ação educativa do enfermeiro, pois ele pode buscar uma negociação com o paciente com base em justificativas para a busca do controle de peso, além de outros hábitos de vida compatíveis com a melhora da saúde. 
A hipertensão arterial é uma das comorbidades que podem acometer a população obesa, podendo relacionar-se também à idade avançada, que foi predominante neste estudo. Dito isso, torna-se essencial que as ações de enfermagem com os idosos hipertensos e seus familiares incluam estratégias de promoção de estilos de vida saudáveis e de encorajamento, fazendo interagir a responsabilidade pessoal na capacitação para o autocuidado e melhoria da qualidade de vida ${ }^{(21)}$.

As pessoas com doenças crônicas, a exemplo da hipertensão arterial e obesidade, sabidamente necessitam de assistência de enfermagem que considere suas especificidades. O enfermeiro é o profissional que possui habilidades para identificar pontos de vulnerabilidade ao cuidado, realizando ações de promoção de saúde, orientação à alimentação e prevenção de ganho de peso, buscando conscientizá-los para a adoção de um estilo de vida compatível com sua qualidade e prevenção de complicações ${ }^{(21)}$.

O diagnóstico de enfermagem Perfusão tissular periférica ineficaz, definido por "redução da circulação sanguínea para a periferia, capaz de comprometer a saúde" ${ }^{(11)}$, foi identificado em $43(33,6 \%)$ sujeitos da pesquisa. Foram enquadrados os indivíduos portadores de doença arterial periférica (associada a outra patologia, que indicou o uso do ACO) e trombose venosa profunda, isoladamente ou em conjunto com outra morbidade, tendo em vista que a TVP prévia é considerada fator de risco para a formação de novos trombos, pois estes apresentam alteração endotelial provocada pelo episódio inicial ${ }^{(1-2)}$.

Quando há risco aumentado de recorrência de eventos embólicos é necessária a manutenção da RNI dentro dos limites terapêuticos com possível ajuste de dose do ACO, buscando a prevenção secundária de novo evento trombótico $^{(1)}$. Já a doença arterial periférica é citada devido à diminuição da irrigação sanguínea para o local acometido, gerando uma perfusão ineficaz dessa área ${ }^{(2-3)}$. Neste escopo, nos cuidados de enfermagem, destacam-se: orientação quanto ao posicionamento do membro, facilitando o retorno venoso, utilização correta de medicações específicas, métodos para alívio e controle da dor e identificação precoce de sinais e sintomas relacionados às complicações secundárias ${ }^{(22)}$.

O diagnóstico de Autocontrole ineficaz da saúde, definido como "padrão de regulação e integração à vida diária de um regime terapêutico para tratamento de doenças e suas sequelas que é insatisfatório para alcançar as metas específicas de saúde" ${ }^{(11)}$ foi identificado em 7 (20\%) sujeitos de pesquisa.

O estudo apontou que 20\% dos sujeitos pesquisados interromperam o uso do ACO por vontade própria. Uma hipótese em relação à suspensão aleatória da terapêutica, sem orientação de um profissional da saúde, é a de que o sujeito não consegue, muitas vezes, identificar a proteção que o medicamento lhe oferece, tendo em vista que as mudanças que geralmente ocorrem estão direcionadas às mudanças em seus hábitos de vida devido ao ACO e não necessariamente por alguma alteração física, mental ou psicológica causada pelo medicamento, o que fica evidente, como é o caso, por exemplo, dos analgésicos, em que o indivíduo consegue notar a diferença antes e depois de ingerir o fármaco, baseando-se na cessação (ou não) da dor.

Esse é mais um ponto de alerta para a importância das orientações e dos cuidados específicos aos usuários do ACO, na intenção de prevenir complicações e atingir os objetivos da terapia para cada grupo, sejam eles usuários temporários ou não desse fármaco. Neste aspecto, reafirma-se a importância da prática sistematizada de enfermagem, incluindo o raciocínio diagnóstico que certamente irá direcionar as ações de cuidado de forma mais robusta e fundamentada ${ }^{(9-10)}$. Não menos importante, como antes problematizado, além da identificação diagnóstica, o enfermeiro precisa capacitar-se na habilidade estratégica, com o intuito de otimizar informações em prol do cuidado individualizado e qualificado.

A descrição do perfil sociodemográfico e clínico de pacientes e seus diagnósticos de enfermagem pode subsidiar o planejamento da assistência aos usuários de ACO, estabelecendo metas individualizadas alcançáveis, e possivelmente contribuindo para a prevenção de complicações 
indesejáveis, como a formação de trombos ou eventos hemorrágicos. Ainda assim, estudos que viabilizem intervenções e avaliações de resultados de enfermagem neste escopo estudado ainda são necessários.

\section{Conclusão}

Foi possível conhecer o perfil sociodemográfico e clínico de pacientes submetidos à anticoagulação oral, acompanhados ambulatoriamente, e os principais diagnósticos de enfermagem elencáveis a tal clientela. Destacaram-se os seguintes achados: perfil de pacientes idosos, casados, com baixa escolaridade, com indicação à terapêutica em estudo principalmente por fibrilação atrial e trombose venosa profunda, em uso de varfarina sódica, e tempo médio de coagulação adequado, expresso por RTI.

Conclui-se que os diagnósticos de enfermagem elencados coadunam-se ao perfil clínico dos usuários, o que reflete um cenário mais focado nas questões orgânico-funcionais, ainda que a necessidade de melhor controle da própria saúde e de domínio da promoção de saúde tenham emergido em menor proporção.

O panorama bem delimitado constatado contribui para a sistematização de cuidados individualizados relacionados especialmente à prevenção de complicações. Neste aspecto, a investigação pode favorecer a consolidação do enfermeiro como gerente do cuidado.

As limitações desta pesquisa relacionam-se ao seu caráter puramente descritivo, não sendo possível estabelecer inferências mais acuradas e/ou generalizações. Todavia, postula-se que o estudo contribui sobremaneira para a prática baseada em evidências de enfermagem, especialmente por ter se debruçado sobre um público peculiar. Logo, a investigação avança no conhecimento para fortalecer a formulação racional de planos de cuidado sistematizados a pacientes em anticoagulação oral em acompanhamento ambulatorial, que normalmente é uma população menos enfocada nos processos de sistematização da assistência e divulgação científica correlata em comparação aos usuários hospitalizados.

\section{Colaborações:}

1. concepção, projeto, análise e interpretação dos dados: Daiana de Freitas, Kelly Ribeiro, Fabiana Gonçalves de Oliveira Azevedo Matos, Ariana Rodrigues da Silva Carvalho e Cláudia Ross;

2. redação do artigo e revisão crítica relevante do conteúdo intelectual: Daiana de Freitas, Kelly Ribeiro, João Lucas Campos de Oliveira, Fabiana Gonçalves de Oliveira Azevedo Matos, Ariana Rodrigues da Silva Carvalho e Cláudia Ross;

3. aprovação final da versão a ser publicada: Daiana de Freitas, Kelly Ribeiro, João Lucas Campos de Oliveira, Fabiana Gonçalves de Oliveira Azevedo Matos, Ariana Rodrigues da Silva Carvalho e Cláudia Ross.

\section{Referências}

1. Guimarães J, Zago AJ. Anticoagulação ambulatorial. Rev HCPA. 2007;25(1):30-8.

2. Leiria TLL, Pellana L, Miglioranza MH, Sant ${ }^{\prime}$ Anna RT, Backer LS, Magalhães E, et al. Varfarina e Femprocumona: experiência de um ambulatório de anticoagulação. Arq Bras Cardiol. 2010;94(1):41-5.

3. Silvestre L, Ministro L, Evangelista A, Pedro LM. Novos anticoagulantes orais no tromboembolismo venoso e fibrilação auricular. Angiol Cir Vasc. 2012 $\operatorname{mar} ; 8(1): 6-11$

4. Maziero VG, Bernardes A, Spiri WX, Gabriel CS. Construindo significados sobre gerência da assistência: um estudo fenomenológico. Ciênc cuid saúde. 2014;13(3):563-70.

5. Soares MI, Resck ZMR, Terra FS, Camelo SHH. Sistematização da assistência de enfermagem: facilidades e desafios do enfermeiro na gerência da assistência. Esc Anna Nery. 2015;19(1):47-53.

6. Horta FG, Salgado PO, Chianca TCM, Guedes HM. Ações de enfermagem prescritas para pacientes internados em um centro de terapia intensiva. Rev Eletr Enf. 2014;16(3):542-8.

7. Soares MI, Resck ZMR, Camelo SHH, Terra FS. Gerenciamento de recursos humanos e sua interface na sistematização da assistência de enfermagem. Enferm Global. 2016;42(3):353-64.

8. Malucelli A, Otemaier KR, Bonnet M, Cubas MR, Garcia TR. Sistema de informação para apoio à 
Daiana de Freitas, Kelly Ribeiro, João Lucas Campos de Oliveira, Fabiana Gonçalves de Oliveira Azevedo Matos, Ariana Rodrigues da Silva Carvalho, Cláudia Ross

Sistematização da Assistência de Enfermagem. Rev bras enferm. 2010;63(4):629-36.

9. Ferreira AM, Rocha EM, Lopes CT, Bachion MM, Lopes JL, Barros ALBL. Diagnósticos de enfermagem em terapia intensiva: mapeamento cruzado e taxonomia da NANDA-I. Rev bras enferm. 2016;69(2):285-93.

10. Matos FGOA, Cruz DALM. Construção de instrumento para avaliar a acurácia diagnóstica. Rev Esc Enferm USP. 2009;43(Esp):1088-97.

11. NANDA-Internacional.Diagnósticos de enfermagem da NANDA, definições e classificação: 2012-2014. Porto Alegre: Artmed; 2013.

12. Corbi ISA, Dantas RAS, Pelegrino FM, Carvalho ARS. Qualidade de vida relacionada à saúde de pacientes em uso de anticoagulação oral. Rev Latino-Am Enfermagem [Internet]. 2011 [cited 2016 Nov 21];19(4):[9 telas]. Available from: http://www. scielo.br/pdf/rlae/v19n4/pt_03.pdf

13. Pelegrino FM, Dantas RAS, Corbi ISA, Carvalho ARS. Perfil sócio demográfico e clínico de pacientes em uso de anticoagulantes orais. Rev Gaúcha Enferm. 2010;31(1):123-8.

14. Carvalho ARS, Ciol MA, Tiu F, Rossi LA, Dantas RAS. Oral anticoagulation: the impact of the therapy in health-related quality of life at sixmonth follow-up. Rev Latino-Am Enfermagem [Internet]. 2013 [cited 2016 Nov 21];21(Spec):[8 telas]. Available from: http://www.scielo.br/pdf/ rlae/v21nspe/14.pdf

15. Henn CB, Rabelo ER, Boaz M, Souza EN. Conhecimento dos pacientes sobre anticoagulação oral crônica acompanhados em ambulatório especializado. Rev Gaúcha Enferm. 2008;29(2):207-13.

16. Ávila CW, Alite GB, Feijó MKF, Rabelo ER. Pharmacological adherence to oral anticoagulant and factors that influence the International Normalized Ratio Stability. Rev Latino-Am
Enfermagem [Internet]. 2011 [cited 2016 Nov 21];19(1):[8 telas]. Available from: http://www. scielo.br/pdf/rlae/v19n1/04.pdf

17. Ansell JE, Hirsh J, Hylek E, Jacobson A, Crowther M, Palaretiet G. Pharmacology and management of the vitamin $\mathrm{k}$ antagonists. American College of Chest Physicians. Evidence-Based Clinical Practice Guidelines (8th Edition). Chest. 2008 Jun;133(6 Suppl):160S-98S.

18. Pedro R, Sobral MA. Novos anticoagulantes orais e risco de hemorragia gastrointestinal: uma realidade? Rev Port Med Geral Fam. 2015;31:286-8.

19. Silva AG, Oliveira AC. Prevenção da infecção da corrente sanguínea relacionada ao cateter venoso central: uma revisão integrativa. Rev Visa Debate. 2016;4(2):117-25.

20. Organização Mundial da Saúde. Diretriz da OMS para a tiragem de sangue: boas práticas em flebotomia [Internet]. Brasília; 2013 [cited 2016 Nov 21]. Available from: www.who.int/injection safety/Phlebotomy-portuges_web.pdf

21. Mártires MAR, Costa MAM, Santos CSV. Obesidade em idosos com hipertensão arterial sistémica. Texto Contexto Enferm [internet]. 2013 [cited 2013 Nov 20];22(3):797-803. Available from: http:// dx.doi.org/10.1590/S0104-07072013000300028

22. Santana CQC, Santos CLO. Identificação do diagnóstico e proposta de intervenção de enfermagem para paciente com trombose venosa profunda. Rev Enferm UFPE Online. 2011 [cited 2016 Nov 21];5(9):2254-9. Available from: http:// www.revista.ufpe.br/revistaenfermagem/index. $\mathrm{php} / \mathrm{revista} /$ article/view/1904

Recebido: 1 de dezembro de 2016

Aprovado: 23 de agosto de 2017

Publicado: 26 de outubro de 2017 